\title{
Robust Image Segmentation by Texture Sensitive Snake Under Low Contrast Environment
}

\author{
Shu-Fai WONG and Kwan-Yee Kenneth WONG \\ Department of Computer Science and Information Systems \\ The University of Hong Kong \\ $\{$ sfwong, kykwong\}@csis.hku.hk
}

Key words: Image Processing Applications, Image Segmentation, Texture Analysis, Medical Image Analysis

Abstract: $\quad$ Robust image segmentation plays an important role in a wide range of daily applications, like visual surveillance system, computer-aided medical diagnosis, etc. Although commonly used image segmentation methods based on pixel intensity and texture can help finding the boundary of targets with sharp edges or distinguished textures, they may not be applied to images with poor quality and low contrast. Medical images, images captured from web cam and images taken under dim light are examples of images with low contrast and with heavy noise. To handle these types of images, we proposed a new segmentation method based on texture clustering and snake fitting. Experimental results show that targets in both artificial images and medical images, which are of low contrast and heavy noise, can be segmented from the background accurately. This segmentation method provides alternatives to the users so that they can keep using imaging device with low quality outputs while having good quality of image analysis result.

\section{Introduction}

Image segmentation is one of the hot topics in the field of image processing and computer vision. Robust image segmentation provides solution to background subtraction and object detection. Recent technology depends heavily on robust object location such that people detection in visual surveillance application, organ detection in computer-aided medical diagnosis and surgery, and background elimination in video compression can be made possible and reliable.

Image segmentation is commonly done by two major approaches, namely pixel intensity analysis and texture analysis. A comprehensive survey on segmentation using intensity analysis can be found in (Pal and Pal, 1993) and those on texture segmentation can be found in (Chellappa and Manjunath, 2001). In pixel intensity analysis, the color intensity or the grayscale level of each pixel will be analysed independently. Segmentation is done by grouping pixels according to the similarity of their intensity value. The major problem of such approach is that it works under the strong assumption of targets with homogeneous intensity value. In addition, analysing pixels inde- pendently is error-prone under non-Gaussian noise model. To relax the assumption and limitation, new segmentation approach using texture was developed. In texture analysis, a texture model is used to describe a region of interest. Patches with similar features in texture model will be grouped together and form a larger patch. Segmentation is then done by grouping of such patches. The major problem in using texture analysis is to find an optimal texture model (e.g. size of the patch, intensity pattern) and this is time consuming and computationally complicated.

In daily applications, images are of low quality and with heavy noise. Medical images, images captured from webcam and images taken under dim light are the examples of such kinds of images. To segment target from such images, traditional segmentation approaches may not be an ideal choice. Segmentation using intensity will be affected by the heavy noise while segmentation by texture will be not robust enough to be used in daily applications.

In this paper, we proposed a supervised texture analysis algorithm that combine the advantage of both intensity analysis and texture analysis to segment target from image with low contrast and heavy noise. The proposed algorithm will learn the edge texture pattern from the image during learning phase. The 
edge pattern can be discovered in the testing image afterward. A snake is then fitted towards the edge. The testing image can then be segmented. The size of the edge texture patch is deterministic as the edge along certain direction will not occupy large number of pixel cells. The pattern can then be learnt and classified as in intensity analysis. The texture patch is used instead of intensity of single pixel, such that the pattern itself is not susceptible to the noise.

\section{System Architecture}

The whole system performs analysis in 2 phases, namely learning phase and testing phase. During the learning phase, both the image and the edge information will be analysed by the system. The system will extract texture feature from the image. The representation of texture feature will be discussed in Section 3. Such feature will be clustered into groups. The groups is then further classified into edge or non-edge texture according to the edge information given. The association between the texture feature and the final group is then established. The clustering algorithm will be explored in Section 4. During testing phase, the texture feature of testing image will be extracted. Finally, classification can be done by comparing the cluster means and the model energy and by applying the cluster association rule. Based on the classification result, foreground can be extracted. The details will be given in Section 5. The flow is summarized in figure 1 .

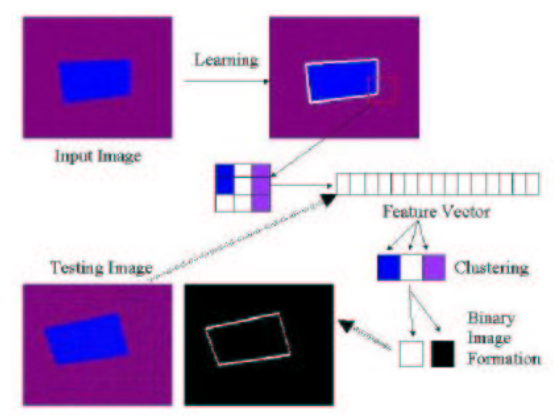

Figure 1: Logic flow of the proposed system.

\section{Texture Analysis by MRF}

Markov Random Field was first developed for texture analysis, e.g. (Cross and Jain, 1983). It can be used to describe a texture and make prediction on the intensity value of a certain pixel given the intensity value of its neighborhood. The theories related to Markov Random Field can be found in (Chellappa and Jain, 1993).

In Markov Random Field, the neighborhood is defined as clique elements. Consider that $S=$ $\left\{s_{1}, s_{2}, \ldots, s_{P}\right\}$ is a set of pixels inside the image, and $N=\left\{N_{s} \mid s \in S\right\}$ is the neighborhoods of the set of pixels. In the system, the neighborhoods are the 8 pixels that with chessboard distance 1 away from the target pixel.

Assuming $X=\left\{x_{s} \mid s \in S\right\}$ is the random variables (the intensity value) for every pixel inside an image, where $x_{s} \in L$ and $L=\{0,1, . ., 255\}$. Besides, we have a class set for texture pattern, $\Omega=$ $\left\{\omega_{S 1}, \omega_{S 2}, \ldots, \omega_{S P}\right\}$ where $\omega_{S i} \in M$ and $M$ is the set of available classes. In the proposed system, we have only two classes, the edge and the non-edge classes.

In Markov chain analysis, the conditional probability of certain pixel being certain class is given by Gibbs distribution according to HammersleyClifford theorem. The density function is $\pi(\omega)=$ $\frac{1}{\sum_{\omega} \exp \left(\frac{-U(\omega)}{T}\right)} \exp \left(\frac{-U(\omega)}{T}\right)$, where $\mathrm{T}$ is the temperature constant, which is used in stimulated annealing. The energy term can be further represented as $U\left(\omega, x_{i}\right)=V_{1}\left(\omega, x_{i}\right)+\sum_{i^{\prime} \in N_{i}} \beta_{i, i^{\prime}} \delta\left(x_{i}, x_{i^{\prime}}\right)$, where $V_{1}\left(\omega, x_{i}\right)$ represents the potential for pixel with certain intensity value belongs to certain class and the $\delta\left(x_{i}, x_{i^{\prime}}\right)$ is the normalised correlation between pixel at $s_{i}$ and those at $s_{i^{\prime}}$.

When the texture is being learnt by the feature learning module, the set of $\beta_{i, i^{\prime}}$ is estimated according to the requirement that the probability of its associated texture class will be maximised. The estimation algorithm used in the system is simulated annealing. The set of $\beta_{i, i^{\prime}}$ corresponds to the correlation value and thus represents the configuration of the pixels such that it can be classified as that texture class. In the system, this set of estimated $\beta$ will be used as texture feature vector. It will be used as input of support vector machine such that the association between texture feature and texture class can be formed.

\section{Texture Clustering using K-Mean Analysis}

$\mathrm{K}$-mean clustering algorithm has been widely used in application that need unsupervised classification. Although there is a learning set in the proposed system, the noise in the image will greatly reduce the reliability of the learning set. To identify those outliers in the learning set, unsupervised clustering will be performed first. The implementation of k-mean clustering algorithm can be found in (Duda et al., 2000).

After performing k-mean clustering on the feature vectors $\beta$, supervised association will be done. Given several clusters after k-mean clustering, some of them correspond to a edge patch with gradient change a along certain direction while the remaining correspond to the non-edge patch, noisy patch or corner patch. Labeling of the cluster is then done according to the association information from the learning 
set. Outliers will be ignored during the labeling stage. Finally, an association between texture feature vector and "edge and non-edge" classes can be formed.

In testing stage, classification can be done simply by extracting the texture feature and then using the association rule formed. Applying such classification scheme on the whole image, a binary map can be formed with ' 1 ' means edge and ' 0 ' means non-edge.

\section{Texture Segmentation by Snake Fitting}

Active contour (Kass et al., 1987) had been used in pattern location and tracking (Blake and Isard, 1998) for a long time. It is good at attaching to object with strong edge and irregular shape. The snake can be interpreted as parametric curve $v(s)=[x(s), y(s)]$. In the proposed system, the initial position of the active contour is the bounding box of the searching window. The active contour will move according to the energy function, $E_{\text {snake }}^{*}=\int_{0}^{1}\left\{\left[E_{\text {int }}(v(s))\right]+\right.$ $\left.\left[E_{\text {texture }}(v(s))\right]+\left[E_{\text {con }}(v(s))\right]\right\} d s$, where $E_{\text {int }}$ represents the internal energy of the snake due to bending, $E_{\text {texture }}$ represents the texture-based image forces, and $E_{c o n}$ represents the external constraint forces. The snake is said to be fitted if the $E_{\text {snake }}^{*}$ is minimised.

The equation is the same as the traditionally used snake-equation but with texture-based image force replaces the original "edge-based" image force. Since the image is of noisy and low contrast, noise will introduce dozens of distracting edges under pixel-based analysis. If the "edge-based" energy is used, the corresponding snake will be highly unstable and inaccurate. Thus, texture-based energy is used in the proposed system. Texture energy is lower near the patch that shows texture changes towards edge texture and thus the snake will shrink towards strong edge in the binary texture binary map described in Section 4 . Texture represents a patch of pixels instead of a single pixel and texture-based analysis is more tolerant to noise compare with pixel-based analysis. Thus, texture-based analysis is a much reliable than edgebased analysis for snake implementation.

\section{Experiment and Result}

The proposed system was implemented using Visual C++ under Microsoft Windows. The experiments were done on a P4 $2.26 \mathrm{GHz}$ computer with 512M Ram running Microsoft Windows.

\subsection{Experiment 1: Artificial Noisy and Low Contrast Images}

In this experiment, the classifier is trained to recognize edge in artificial images with heavy noise and of low contrast. A texture-sensitive snake is then fitted towards the texture-edge from the initial position close to the real edge. The result is shown in figure 2 . It shows that the binary image (texture map) match the edge quite well. The snake can fit toward the edge quite well too. The relative root-mean-square error (i.e. the relative distance between the control points and the real edge) is less than $5 \%$ when comparing with ground truth images. The processing time is around 10s where the images with average size 267 x 255 pixels.
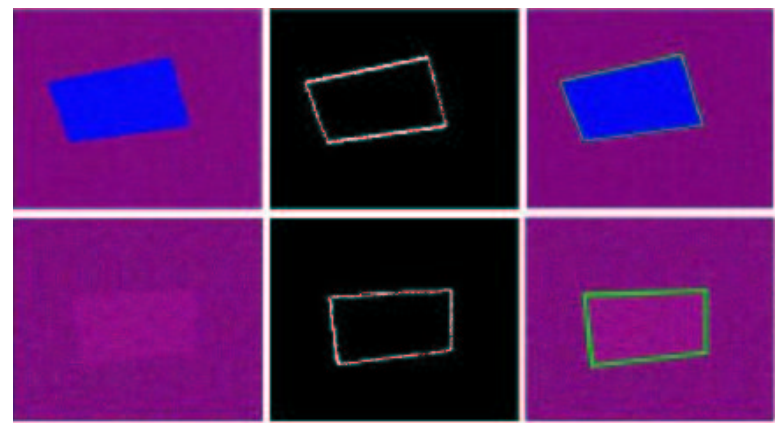

Figure 2: The first row shows the segmentation result of using noisy image while the second row shows the result of using low contrast image. The left-most images are the testing images. Middle images are the corresponding binary map after final classification. The right-most images show the result of snake-fitting.

\subsection{Experiment 2: Low Contrast and Noisy Medical Image}

In this experiment, the backbone has to be segmented from the medical image with poor quality and low contrast. Actually, the image may not be segmented easily manually. The result of segmentation is showed in figure 3. It shows that the snake can fit some of the backbones very well. The accuracy cannot be determined here due to no ground truth image provided. The processing time is around $18 \mathrm{~s}$ where the image with size $600 \times 450$ pixels.
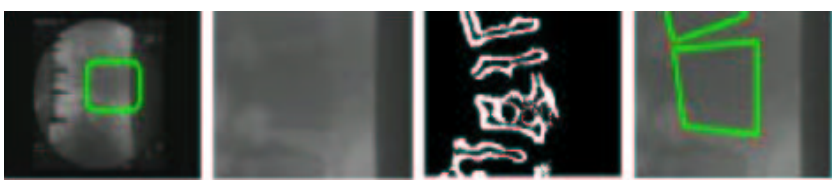

Figure 3: The left-most image is the given medical image. The second left image shows enlarged portion of the first image. The third image is the corresponding binary map. The last image is the result of snake-fitting. 


\subsection{Experiment 3: Comparison with other approaches}

In this experiment, pixel-based analysis, texturebased analysis and traditional snake-fitting are performed on images used in experiment 1 and 2 . The results are shown in figure 4 , figure 5 and figure 6 respectively. From the results, it seems that the proposed method performs better than these approaches. Pixel-based analysis cannot perform well without knowing the optimal similarity-tolerant level. In texture-based analysis, color patches extracted are not semantically connected (i.e. color patches can be corners, edges and heterogeneous region) and there are so many clusters (in black color) are unclassified due to the noisy nature. Traditional snake approach is sensitive to cluttered background and image noise.
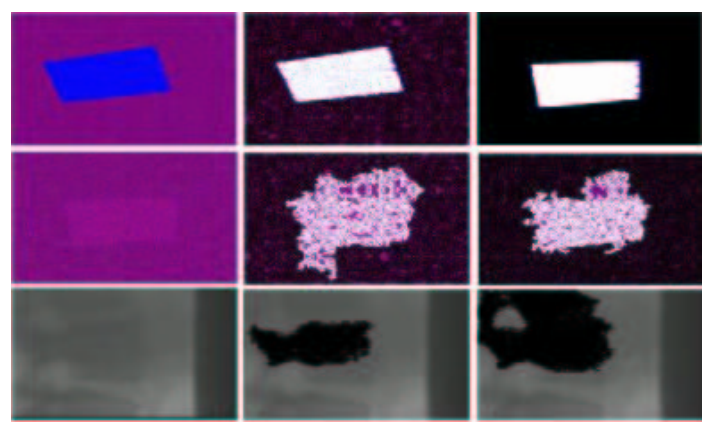

Figure 4: The result of applying pixel-based analysis is shown. These rows show the result of using artificial noisy image, low contrast image and medical image respectively. The images on first column are the testing images, the images on the second column are resultant images on low similarity-tolerant level, while images on the third column are resultant images on high similarity-tolerant level. The two largest clusters consists of pixels with similar attributes are indicated by black and white. Those unclassified and small pixel patches are kept in original color.

\section{Conclusions}

There is a demand of segmenting a target from images with low contrast and heavy noise in application like medical imaging. However, commonly used image segmentation approach can only work properly if the input image has homogeneous intensity or texture of high quality. The proposed segmentation algorithm aims at segmenting a target from an image of low quality by texture pattern extraction and clustering. To have better and more accurate segmentation result, a snake is fitted towards to edge pattern such that the boundary of the target can be captured. It combines the advantages of using pixel analysis and texture analysis such that it can segment the target from an image of low quality in the experiments.

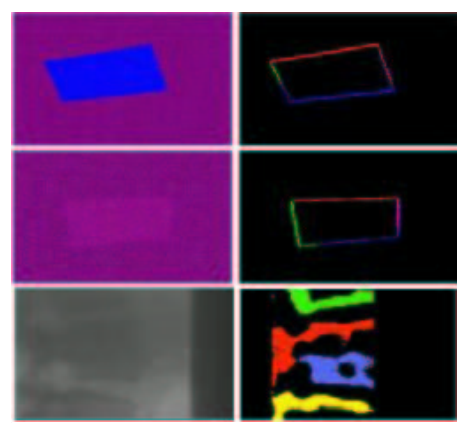

Figure 5: The result of applying texture-based analysis is shown. The rows are ordered in the same way as in Figure 4. The images on first column are the testing images while those on second column are the resultant image. Patches with similar texture are grouped together and form a color cluster. Those small patches cannot be grouped together are indicated by black color.
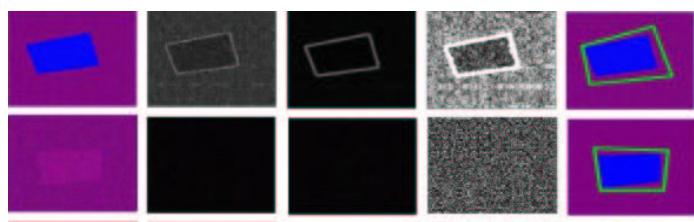

5
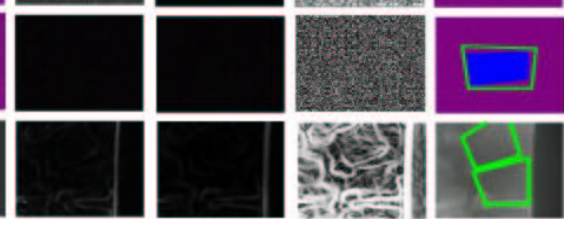

Figure 6: The results of using snake approach are shown. The rows are ordered in the same way as in Figure 4. The first column shows the input image. The second column shows the edge detection result on unsmoothed input image while the third column shows the edge detection result on smoothed input image. The fourth column shows the equalized result of the resultant image on the third column. The last column shows the snake applied on the the input image with reference to the edge map on the third column.

\section{REFERENCES}

Blake, A. and Isard, M. (1998). Active Contours. Springer. Chellappa, R. and Jain, A. (1993). Markov Random Fields: Theory and Applications. Academic Press.

Chellappa, R. and Manjunath, B. (2001). Texture classification and segmentation. In FIU01, page Chapter 8.

Cross, G. and Jain, A. (1983). Markov random field texture models. PAMI, 5(1):25-39.

Duda, R. O., Hart, P. E., and Stork, D. G. (2000). Pattern Classification. John Wiley and Sons, Inc., second edition.

Kass, M., Witkin, A., and Terzopoulos, D. (1987). Snakes: Active contour models. In Proc. Int. Conf. on Computer Vision, pages 259-268.

Pal, N. and Pal, S. (1993). A review on image segmentation techniques. $P R, 26(9): 1277-1294$. 